\title{
A smart collision recovery receiver for RFIDs
}

\author{
Jelena Kaitovic ${ }^{*}$, Robert Langwieser and Markus Rupp
}

\begin{abstract}
In this work, we focus on framed slotted Aloha (FSA) and passive ultra high-frequency radio frequency identification multi-antenna systems with physical layer collision recovery. We modify the tags slightly by adding a so-called 'postpreamble' that facilitates channel estimation. Furthermore, we investigate the throughput performance of advanced receiver structures in collision scenarios. More specifically, we analyse the throughput of FSA systems with up to four receive antennas that can recover from a collision of up to eight tags on the physical layer and acknowledge all tags involved in that collision. Due to the higher collision recovery capabilities, the frame sizes can be significantly reduced, and thus, the throughput can be increased. We also derive analytically optimal frame sizes, given that a certain number of collisions can be resolved. We further study the constraints to the throughput due to the structure of our receiver and channel estimation for different collision scenarios. Furthermore, we propose a novel collision recovery method with two phases: first, a successive interference cancellation and, second, a projection of the constellation into the orthogonal subspace of the interference. Additionally, the inventory time, i.e. the number of slots necessary to successfully decode all tags in the reader range, is calculated and compared for different receiver types. A validation of our theoretical predictions is achieved by means of simulations. We show that by our proposed methods, we can realistically achieve more than ten times higher throughput or, equivalently, a reduction of the inventory time by more than $90 \%$.
\end{abstract}

\section{Introduction}

Usually, several radio frequency identification (RFID) tags operate within a coverage area of an RFID reader. For the efficient scheduling of tag transmissions, framed slotted Aloha (FSA) or binary tree protocols are used on the medium access control layer. Our focus is on passive ultra high-frequency (UHF) RFID systems and FSA as defined in the EPCglobal standard [1]. If multiple tags respond simultaneously, a collision at the air interface occurs. The standard collision detection mechanism regards this as a destructive event and discards the information. Thus, only slots in which one tag is active can be decoded successfully [2]. This determines the maximal throughput per slot for an FSA system. The maximum throughput value of 0.368 is achieved when the inventory frame size $F$ is equal to the tag population size $N$.

\subsection{Related work}

To overcome such limitations, different research groups are working on collision arbitration protocols and

*Correspondence: jelena.kaitovic@nt.tuwien.ac.at

Institute of Telecommunications, Vienna University of Technology,

Gusshausstrasse 25/389, Vienna 1040, Austria collision recovery procedures. Knerr et al. in [3] formulated a maximum likelihood estimator to yield the estimated number of tags on a slot-wise basis. Their method can be applied for an immediate update of the frame size, during the frame duration, according to the probability level of the current slot-by-slot estimate. Yu et al. in [4] proposed an anti-collision algorithm based on smart antenna technology, which leads to the use of the space division multiple access in RFID systems. They divided the reader coverage area into several subspaces and used an FSA or a binary tree search in each sector, but they did not recover from collisions. If readers with collision recovery (CR) features are available, slots with colliding tags can also be decoded successfully, and the throughput increases further. Knowing the maximum number of collisions that can be resolved by a certain receiver architecture, the frames can be reduced which results in further throughput enhancement and smaller inventory times. A practically working $C R$ on a physical layer with a single antenna reader receiver with two colliding tags in one slot is demonstrated in [5]. With such reader, Angerer et al. obtained an expected throughput increase of approximately $60 \%$. In [6,7], the authors derived a single antenna detection scheme for the simultaneous transmission of

\section{是 Springer}

(C) 2013 Kaitovic et al.; licensee Springer. This is an Open Access article distributed under the terms of the Creative Commons Attribution License (http://creativecommons.org/licenses/by/2.0), which permits unrestricted use, distribution, and reproduction in any medium, provided the original work is properly cited. 
two tags using a memory-assisted detection of collided FM0 signals. Furthermore, they calculated an inventory time reduction of $8 \%$ to $17 \%$ when a two-tag detection and collision recovery is utilized. In [8], De Donno et al. showed, by experiments, that performances of conventional RFID systems can be considerably enhanced by using collision recovery in case of two colliding tags. The authors achieved an inventory time reduction of $26 \%$ in actual measurements taken with a software-defined RFID reader and off-the-shelf programmable tags. In [9], Kim et al. presented an improved binary tree collision arbitration protocol that decreases the number of retransmissions and reduces the identification delay by exploiting multiple antennas at the reader. Theoretical calculations of the FSA system throughput for the reader with physical layer collision recovery were performed in [10], and a significant increase is shown. Additionally, the authors proved a single antenna receiver with a channel estimation procedure for recovering from a two-tag collision to work in practice. Moreover, they have shown that multiple antenna receivers with perfect channel knowledge are capable of recovering from a collision of $R \leq M$ tags. Here, $M$ denotes the so-called collision recovery factor, that is the maximum number of colliding tags a reader can resolve under best circumstances. It is directly related to the number of receive antennas $N_{\mathrm{RA}}$. In [10], it was conjectured that $M=N_{\mathrm{RA}}$. In [11], the single antenna receiver was further extended, and a 2.6-fold throughput increase compared to a conventional reader was achieved with four receiving antennas. Such reader is capable of recovering from a collision of $R \leq M=2 N_{\mathrm{RA}}$ tags in a slot and to acknowledge one out of them. Such perfect CR scheme comes with a caveat, though: at least a moderate channel estimate for each tag is required. Since a tag signal, modelled according to EPCglobal, is not suitable for channel estimation in collision scenarios, in [12], we have proposed a modified tag response that can be used for channel estimation. This modification requires minor changes in the standard by adding a so-called 'postpreamble' sequence. A collision recovery from a scenario in which all tags involved in the collision have different, unique 'postpreambles' is considered, and excellent channel estimation results are obtained. Moreover, in [12], we have identified a potential throughput increase of more than five times in the case of a reader that can recover from a collision of up to eight tags in one slot and acknowledge two of them. Fyhn et al. showed that channel fading, the difference in delay and the tag frequency dispersion can be used for easier separation of colliding signals in a multipacket receiver in [13]. In [14], Ricciato et al. achieved a $20 \%$ to $25 \%$ gain in throughput by applying inter-frame successive interference cancellation (ISIC) with respect to traditional intra-frame SIC. Myung et al. elaborated frameless binary splitting methods in [15].
Their results show that adaptive binary splitting reduces delay and tag communication overhead for the tag reading process. In [16], a theoretical study on collision recovery binary tree algorithm is proposed, and a closed form for calculating system efficiency is derived.

\subsection{Contribution}

This work is focused on the analysis of the theoretical throughput of an FSA system and its associated constraints. In this paper, we investigate the influence of a tag signal modification by adding postpreambles, we analyse the receiver structure and $\mathrm{CR}$ properties and compare obtained results to their theoretical throughput. The collision recovery is performed with receivers that perform channel estimation using a small $(C=8)$ set of postpreambles. Since the distribution of the postpreamble in the colliding tags is random, different collision scenarios need to be considered. In this contribution, we deviate from some optimal prior assumptions and study the performance on more feasible constraints, including a fixed set of postpreambles, additive noise impacting the channel estimation and collision recovery as well as collision detection algorithms with limited quality. We compare our results with the performance of an EPC protocol compliant reader (so-called conventional reader).

The remainder of the paper is organized as follows: Section 2 provides a brief introduction into the mathematical modelling of RFID transmissions and provides a basis for our advanced algorithms. The theoretical throughput of FSA systems and its associated constraints are analysed in Section 3. In Section 4, we introduce a recovery procedure from a collision in which two tags use the same postpreamble and explain our two-step recovery approach. Section 5 provides a performance analysis by means of simulations where we validate our previous predictions, and the last section concludes the paper.

\section{RFID multi-antenna reader}

Figure 1 depicts a typical communication between a single reader equipped with one transmit and $N_{\text {RA }}$ receive antennas, and $N$ tags. In passive RFID systems, a tag is powered by the reader with energy in the form of a continuous carrier transmission. For communicating with the reader, tags apply backscatter modulation. In $[17,18]$, the authors proposed a two-way Rician channel model for RFID scenarios based on carried out channel measurements. They also showed that since the Rician factor strongly depends on the environment, a better fit to the measurement data was achieved by applying a double Rayleigh distribution. Thus, we assume that our channel is a double Rayleigh fading channel and is modelled as a multiplication of a forward channel $h_{j}^{f}$ and a backward channel $h_{i, j}^{b}$ as explained in [11,19]. In case when $R$ tags 


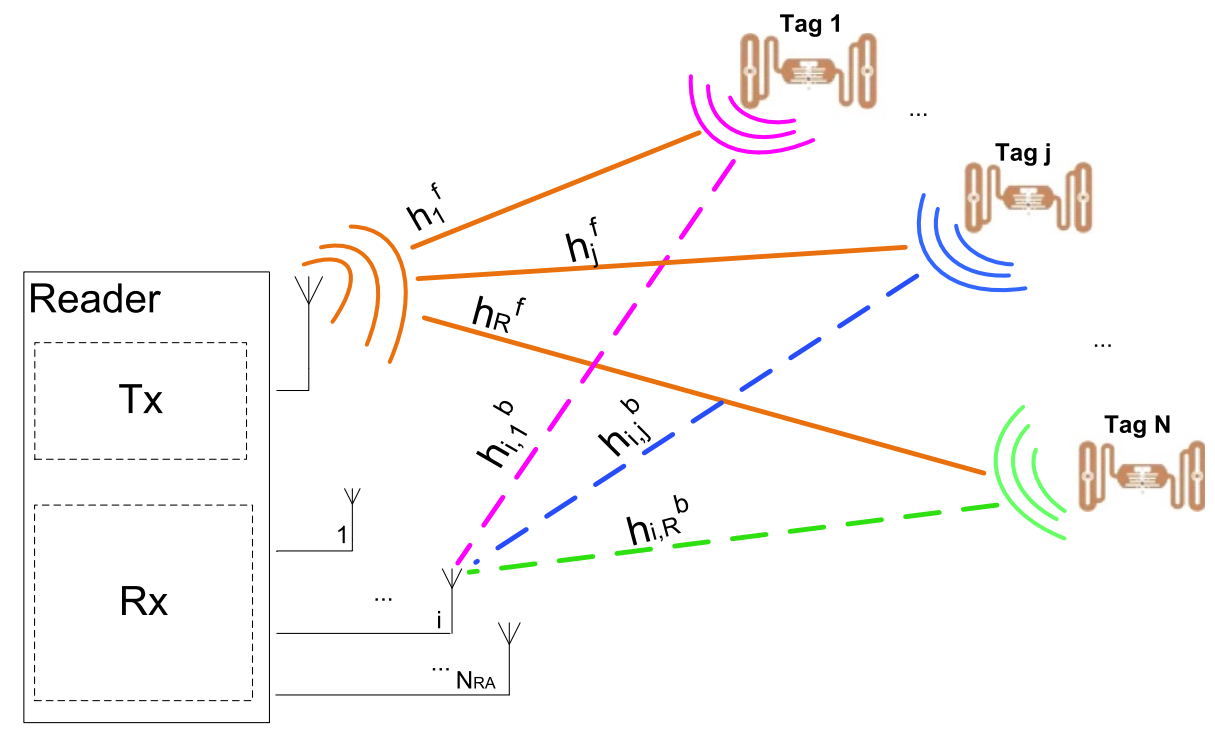

Figure 1 Communication between a single reader equipped with one transmit and $N_{\mathrm{RA}}$ receive antennas, and $N$ tags [12]. With permission of the IEEE.

respond simultaneously in one slot, the baseband signal at the receive antenna $i$ is as follows:

$$
r_{\mathrm{c}, i}(t)=\sum_{j=1}^{R} h_{j}^{f} h_{i, j}^{b} a_{j}(t)+n_{i}(t), \quad i=1, . ., N_{\mathrm{RA}},
$$

where $r_{\mathrm{c}, i}(t)$ is the complex-valued received signal, $n_{i}(t)$ is the noise at the $i$ th antenna, and $a_{j}(t)$ denotes the modulation signal of tag $j$. Here, we assume that the transmit and receive parts of a reader are perfectly isolated (there is no carrier leakage). Since all signals, except the tag modulation signal, are complex values, we can double the number of equations by splitting Equation (1) in real $\Re\{\cdot\}$ and imaginary part $\Im\{\cdot\}$. This allows the recovery from a collision of $R \leq M$ tags, where $M=2 N_{\mathrm{RA}}$ denotes the collision recovery factor.

For collision recovery using multiple receive antennas, a minimum mean square error (MMSE) receiver is proposed in [11]. The signal at the output is as follows:

$$
\hat{\mathbf{a}}_{\mathrm{MMSE}}(t)=\left(\hat{\mathbf{H}}^{T} \hat{\mathbf{H}}+\sigma_{n}^{2} \mathbf{I}_{R}\right)^{-1} \hat{\mathbf{H}}^{T}(\mathbf{r}(t)-\hat{\mathbf{H}} \overline{\mathbf{a}}(t))
$$

where $\hat{\mathbf{H}}=\left[\Re\left\{\hat{\mathbf{H}}_{\mathbf{c}}\right\} \Im\left\{\hat{\mathbf{H}}_{\mathbf{c}}\right\}\right]^{T}$ is the $2 N_{\mathrm{RA}} \times R$ estimated real-valued channel matrix, and $\hat{\mathbf{H}}^{T}$ denotes its


the $2 N_{\text {RA }} \times 1$ real-valued column vector of the received signal, $\mathbf{I}_{R}$ denotes the $R \times R$ identity matrix, $\sigma_{n}^{2}$ is the noise power and $\overline{\mathbf{a}}(t)=E\{\mathbf{a}(t)\}=\frac{1}{2}$ due to the on-off keying modulation. For convenience, in Table 1, an overview of the most important parameters and terms in this paper is listed. There, small bold terms indicate vectors, while those in capital indicate matrices.

According to the EPCglobal standard for UHF RFID [1], a tag response to the Query command is composed of a preamble and an RN16 signature. Since all tags use the

\begin{tabular}{|c|c|}
\hline Variable & Description \\
\hline$N_{\text {RA }}$ & Number of receiving antennas \\
\hline$M=2 N_{\mathrm{RA}}$ & $\begin{array}{l}\text { Collision recovery factor / number of } \\
\text { tags the reader is capable of resolving }\end{array}$ \\
\hline J & $\begin{array}{l}\text { Maximum number of tags } \\
\text { the reader acknowledges }\end{array}$ \\
\hline$J_{C}$ & $\begin{array}{l}\text { Maximum number of tags with } \\
\text { colour } C \text { the reader acknowledges }\end{array}$ \\
\hline$R \in[0 . . N]$ & Number of tags active in the same slot \\
\hline$R_{C} \in\left[0 . . \frac{N}{C}\right]$ & $\begin{array}{l}\text { Number of tags per slot } \\
\text { with colour } C\end{array}$ \\
\hline$j \in[1 . . R]$ & Tag index per slot for $R>0$ \\
\hline$i \in\left[1 . . N_{R A}\right]$ & Receive antenna index \\
\hline $\mathbf{r}_{C}(t)$ & Received signal vector $\in \mathbb{C}^{N_{\mathrm{RA}} \times 1}$ \\
\hline $\mathbf{H}_{\mathbf{c}}$ & Channel matrix $\in \mathbb{C}^{N_{\mathrm{RA}} \times R}$ \\
\hline $\mathbf{a}(t)$ & Modulation vector $\in \mathbb{R}^{R \times 1}$ \\
\hline N & Number of tags within the reader range \\
\hline F & Number of slots in a frame \\
\hline$r_{i}[k]$ & $\begin{array}{l}\text { Sample of the received signal from ith antenna } \\
\text { taken within duration of the first preamble bit } t_{1 \text { bit }}\end{array}$ \\
\hline s/ & Collision scenario $/=1,2, \ldots, S(R)$ \\
\hline
\end{tabular}

Table 1 Most commonly used symbols and parameters 
Table 2 Set of postpreambles [12], with permission of the IEEE

\begin{tabular}{ll}
\hline Sequence & Postpreamble \\
\hline $\mathbf{p}_{1}$ & 1010101010101010 \\
$\mathbf{p}_{18}$ & 1010101101010100 \\
$\mathbf{p}_{69}$ & 1011010101001010 \\
$\mathbf{p}_{86}$ & 1011010010110100 \\
$\mathbf{p}_{171}$ & 1101001011010010 \\
$\mathbf{p}_{188}$ & 1101001100101100 \\
$\mathbf{p}_{239}$ & 1100110100110010 \\
$\mathbf{p}_{256}$ & 1100110011001100 \\
\hline
\end{tabular}

same preamble and the RN16 is a 16 bit-random number, it cannot be used for channel estimation in a general multi-user setup. To overcome this hurdle, in [12], we proposed a modification of the tag response by adding a postpreamble. The set of the postpreambles is shown in Table 2. As explained in [12,20], postpreambles of colliding tags are designed to form a set of mutually orthogonal sequences $\mathbf{S}_{\mathrm{M}}$ so that optimal channel estimates are obtained using a least squares (LS) estimator [12]:

$$
\hat{\mathbf{H}}_{c}=\mathbf{r}^{\mathrm{pP}}(t) \cdot \mathbf{S}_{\mathrm{M}}^{H}\left(\mathbf{S}_{\mathrm{M}} \mathbf{S}_{\mathrm{M}}^{H}\right)^{-1}
$$

Here, $\mathbf{r}^{\mathrm{pP}}(t) \in \mathbb{C}$ denotes the part of the received signal containing the postpreamble.

\section{FSA with CR}

FSA is an interrogation scheme, and it is used for scheduling the transmission of tags. Our focus is on FSA as defined in the second-generation EPCglobal standard for passive UHF RFID [1]. Through the Query command, the reader announces the beginning of the frame and the frame duration (number of slots in a frame). Tags randomly choose slots for transmission. For a conventional reader without $\mathrm{CR}$, only slots without a collision can be decoded successfully, and it is well known that the maximal throughput is achieved when the frame size $F$ is the same as the tag population size $N$. If more than one tag is active in one slot, a collision at the air interface occurs and the entire slot is discarded. With CR capable readers, and some changes in the protocol, it is possible to use the information from slots with a collision to increase the throughput together with shorter frame sizes.

In order to evaluate the performance of FSA systems with $\mathrm{CR}$, theoretical bounds are calculated in the following. These bounds are determined by the receiver structure, the tag signal modification and the channel estimation. Furthermore, we denote the number of simultaneously acknowledged tags as $J$. The maximum number of $J$ is given by $M$, but $J$ can also be limited by the standard (the current standard only allows for $J=1$ ) and/or the capabilities of the receiver technique. By introducing such a variable $J$, we can study beforehand the expected throughput improvements and later compare with the true achievable values of $J$ based on our receiver capabilities.

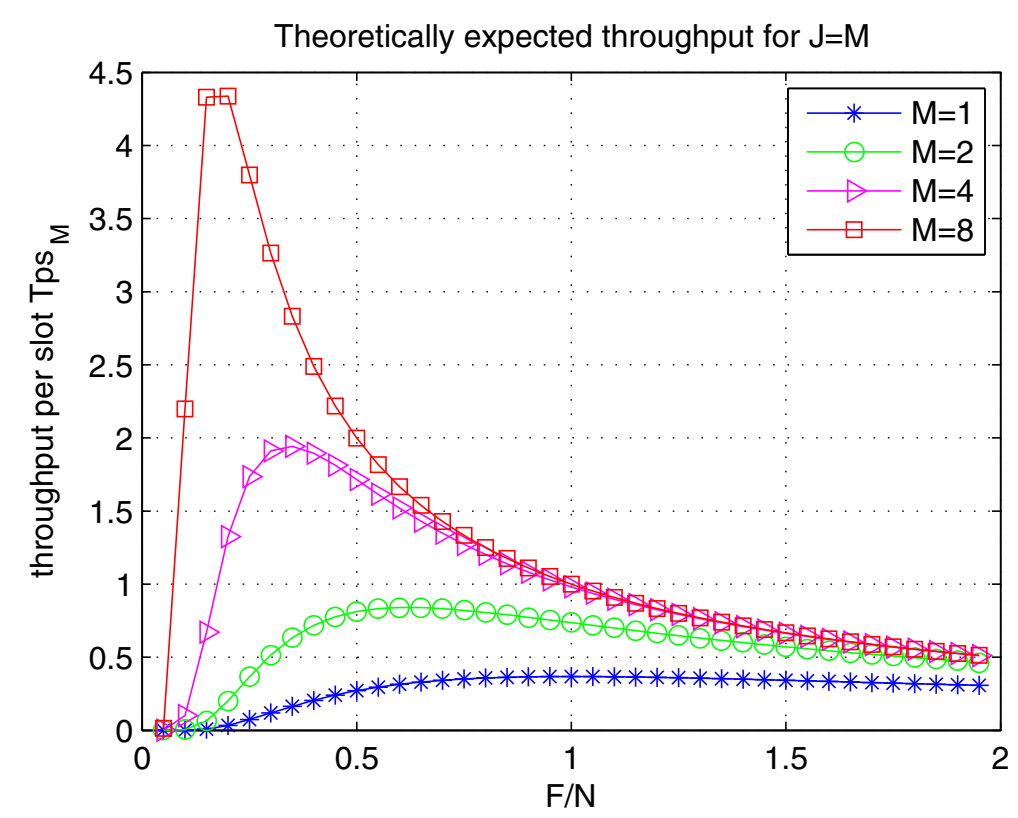

Figure 2 Theoretically expected throughput per slot curves constrained with receiver structure. Expected throughput as a function of slots per tag population $F / N$ for $J=M=2 N_{R A}$ acknowledgements following Equation (4) [21], with permission of the IEEE. 
Table 3 Maximal theoretical throughput per slot constrained with receiver structure [21], with permission of the IEEE

\begin{tabular}{lccc}
\hline System & $\boldsymbol{F}_{\text {opt }} / \boldsymbol{N}$ & Tps & $\boldsymbol{R}_{\text {Tps }}$ \\
\hline$M=1 \quad J=1$ & 1 & 0.368 & 1.000 \\
$M=2 \quad J=2$ & 0.618 & 0.841 & 2.285 \\
$M=4 \quad J=4$ & 0.339 & 1.944 & 5.283 \\
$M=8 \quad J=8$ & 0.173 & 4.479 & 12.171 \\
\hline
\end{tabular}

The table shows the optimal ratio $F_{\text {opt }} / N$, maximal theoretical throughput per slot constrained with receiver structure and its relative improvement (see Appendix 1 for more details).

\subsection{Throughput constrained with receiver structure}

The maximal theoretical throughput per slot, obtained for an optimal reader that can recover from a collision of $R \leq$ $M=2 N_{\text {RA }}$ tags and can acknowledge $(J=M)$ in the case of perfect channel knowledge [21], reads as follows:

$$
\operatorname{Tps}_{M}=\sum_{R=1}^{M}\left(\begin{array}{l}
N \\
R
\end{array}\right)\left(\frac{1}{F}\right)^{R}\left(1-\frac{1}{F}\right)^{N-R} R \text {. }
$$

Figure 2 shows successfully read tags per slot with respect to the normalized frame size. The throughput curves follow with very good agreement a simple function in the ratio $F / N$ (see Appendix 1 for more details). The maximal theoretical throughput per slot Tps for a reader with different collision recovery factor $M$ is listed in Table 3. This maximum is obtained for the optimal frame size $F_{\text {opt }}$, which is normalized by the tag population size $N$. Furthermore, for each case, the relative improvement $R_{\mathrm{Tps}}$ in Tps with respect to a conventional system with $C=1$, $J=1$ is listed.
By assuming an optimal receiver structure and a tag population of $N=1,000$, the maximal theoretical throughput per slot is 4.479 , achieved for a frame that contains only 173 slots. However, the values obtained here are in the case of perfect channel knowledge, which is much higher than we can expect to achieve with a feasible receiver. In order to recover from a collision when applying an MMSE receiver (Equation (2)), we need to estimate the channel. For channel estimation, we introduce additional postpreambles. In the following paragraph, we investigate the influence of the tag signal modification to the system throughput.

\subsection{Throughput constrained with postpreambles}

Assume that the tag response to the Query command has been modified according to [12]. As explained there, a postpreamble is added in order to support channel estimation, and the desired case is that all tags involved in a collision have orthogonal postpreambles to distinguish them. For easier explanation, we will use the term 'tags with different colours' to denote the tag's property of having different postpreambles. If we assume a uniform distribution of postpreambles between tags in the population, we can view the tag population of size $N$ as $C$ partitioned tag populations, each with the average size $N / C$, where $C$ is the number of different colours (number of different postpreambles in a set) as shown in Figure 3. Theoretically, as long as there is a tag of unique colour among the active tags in a slot, we can differentiate the related signal and acknowledge this tag. Even if there are two tags with the same colour involved in a collision, we expect to recover from that particular collision and to acknowledge both tags by applying the projection method proposed in [10].

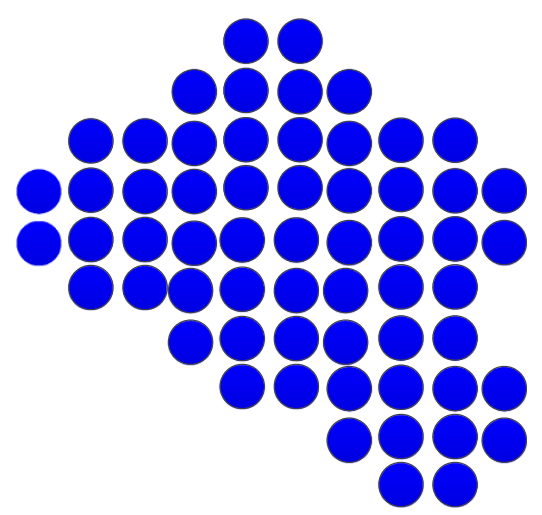

tag population $N$
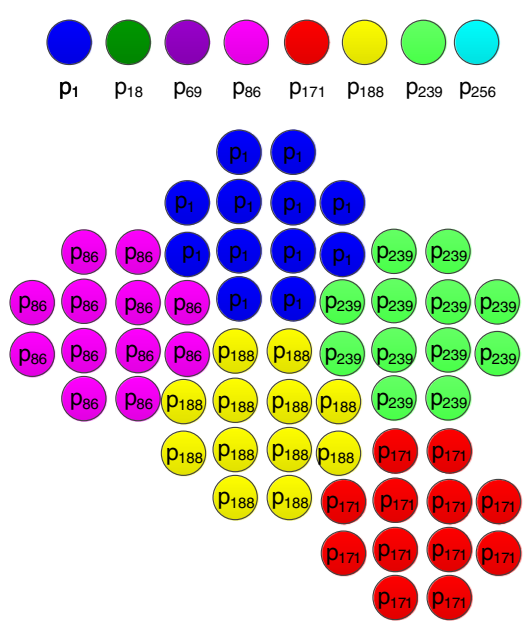

$C$ partitioned tag populations $N / C$

Figure 3 Tag population $N$ (left) and C partitioned populations with N/C tags each (right). 


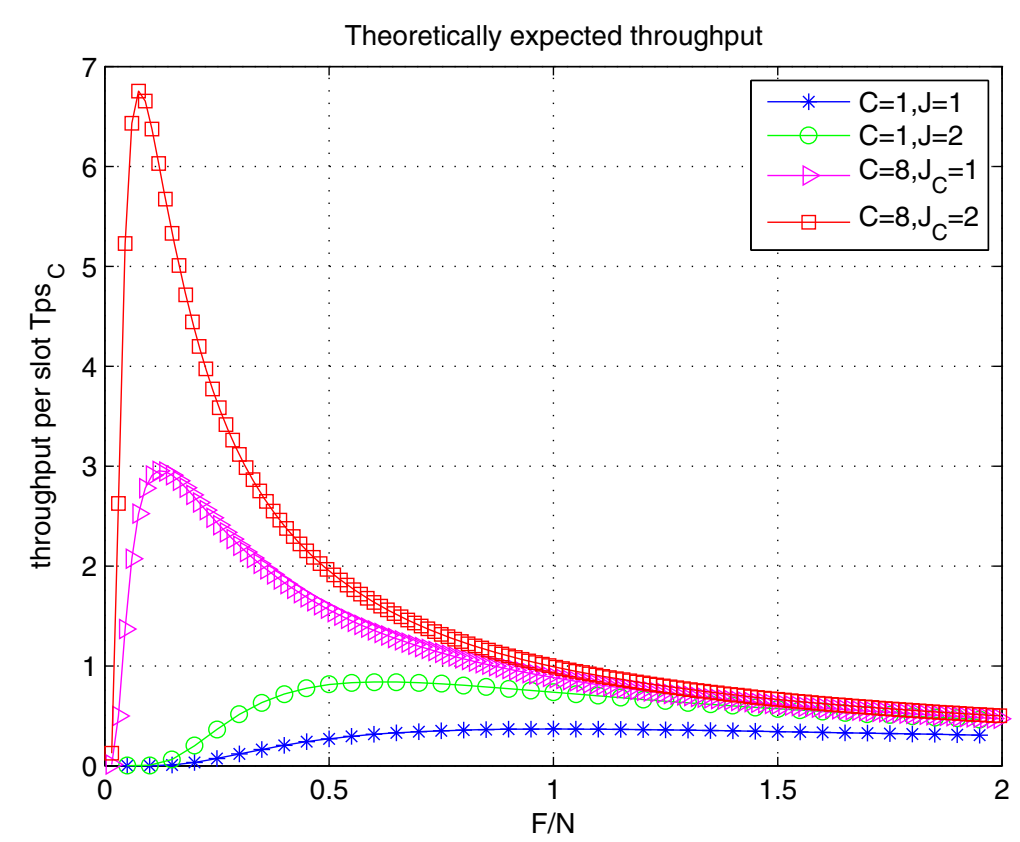

Figure 4 Theoretically expected throughput per slot curves constrained with postpreambles. Expected throughput as a function of slots per tag population $F / N$ for $C=\{1,8\}$ colours and $J=\{1,2\}$ acknowledgements following Equation (5).

Now, under the assumption that we can acknowledge up to $J_{C}$ tags with identical colour per slot, given the tag population size $N$, the frame size $F$ and $C$ different postpreambles in a set, the Tps is as follows:

$$
\operatorname{Tps}_{C}=C \sum_{R_{C}=1}^{J_{C}}\left(\begin{array}{c}
\frac{N}{C} \\
R_{C}
\end{array}\right)\left(\frac{1}{F}\right)^{R_{C}}\left(1-\frac{1}{F}\right)^{\frac{N}{C}-R_{C}} R_{C}
$$

where $R_{C}$ denotes the number of tags per slot with identical colour.

Figure 4 shows the theoretically expected throughput per slot curves. The throughput curves follow with very good agreement a simple function in $F / N$ (see Appendix 2 for more details). The first two curves, blue and green, are simulated for a tag population size of $N=1,000$ according to Equation (4) for $J=M$, while the magenta and red curves are calculated with the partitioned tag population of size $C \times \frac{N}{C}=8 \times 125$, Equation (5). The conventional receiver that can deal with just one tag transmitting per slot and acknowledge that single tag $(J=1)$ is presented by the blue curve. Here, the theoretical maximum throughput per slot is 0.368 and is achieved for the frame size equal to the tag population size. The green curve represents a receiver that can recover from a collision of two tags and acknowledges both signals $(J=2)$. In this case, the maximal throughput per slot is obtained for a shorter frame size and is 0.841 . Due to the shortening of the frame by $38.2 \%$ (see Table 4 ), the overall throughput is further increased by $2.285 / 0.618=3.697$. For the next two curves, we assume to have $C=8$ different partitions of tags. For the magenta curve, eight different partitions of tags are in the reader range, and the throughput benefits just from the existence of tags with single unique colours that are correctly acknowledged $\left(J_{C}=1\right)$, while the red curve represents also a throughput increase due to the recovery from a collision of pairs of colours $\left(J_{C}=2\right)$. Here, the throughput is further increased; for $C=8$ and $J_{C}=1$, the relative increase is $8.030 / 0.125=64.24$, while for $C=$ 8 and $J_{C}=2$, it is even $18.361 / 0.077=238.45$. However, due to the additional postpreamble, also a slot duration increase of $26.67 \%$ needs to be taken into account.

Table 4 shows the optimal values of the frame size normalized by $N$, the corresponding maximum Tps, as well as the relative improvement in Tps with respect to a conventional system with $C=1, J=1$. The last column labelled $R_{\mathrm{Tpf}}$ lists the relative improvement $R_{\mathrm{Tpf}}$ in throughput per

Table 4 Maximal theoretical throughput per slot constrained with postpreambles

\begin{tabular}{lcccc}
\hline System & $\boldsymbol{F}_{\text {opt }} / \boldsymbol{N}$ & Tps & $\boldsymbol{R}_{\text {Tps }}$ & $\boldsymbol{R}_{\text {Tpf }}$ \\
\hline$C=1 \quad J=1$ & 1 & 0.368 & 1.000 & 1.000 \\
$C=1 \quad J=2$ & 0.618 & 0.841 & 2.285 & 3.697 \\
$C=8 \quad J_{C}=1$ & 0.125 & 2.955 & 8.030 & 47.096 \\
$C=8 \quad J_{C}=2$ & 0.077 & 6.757 & 18.361 & 174.824 \\
\hline
\end{tabular}

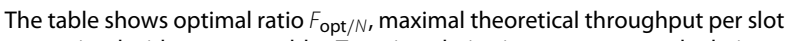
constrained with postpreambles $\operatorname{Tps}_{C}$, its relative improvement and relative improvement in throughput per frame (see Appendix 2 for more details). 
frame with respect to a conventional system with $C=1$, $J=1$ calculated as follows:

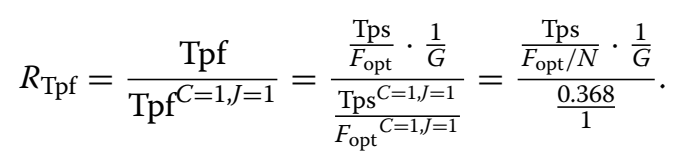

Here, we take into account shorter frames $\left(F_{\text {opt }}\right)$ and the stretching of the frames by an additional postpreamble $(G)$ that is required for $C>1$ colour. The signal of a tag consists of a preamble (6 bits), a postpreamble (8 bits) and an RN16 (16 bit), and each bit is encoded with FM0. Thus, the frame stretching factor is $G=\frac{6+8+16}{6+16}=1.364$. In spite of the small loss due to $G$, the relative improvement $R_{\mathrm{Tpf}}$ can climb up to 174.8 . However, such considerations are too optimistic as the following example shows. For $N_{\mathrm{RA}}$ receive antennas, only $R \leq M=2 N_{\mathrm{RA}}$ tags can be resolved. Consider, for example, a scenario in which each of the $C=8$ colours appears twice. Then, there are in total $R=C \cdot R_{C}=16$ tags in this slot, but with $N_{\mathrm{RA}}=4$, only eight tags can be resolved. Practically, Equation (5) needs at least to be constrained by $C \cdot J_{C} \leq M$. Furthermore, the channel cannot be estimated in all collision scenarios. We thus derive tighter bounds in the following.

\subsection{Expected throughput for covered scenarios}

Since colliding tags have randomly distributed postpreambles, several collision scenarios are possible [21]. It can happen that all tags involved in a collision have different, unique postpreambles $(1+1+\ldots+1)$, and in that case, we can estimate the channel with a regular LS estimator. However, this scenario is more rare when more tags are involved in a collision. In Table 5 , all scenarios are listed for up to eight tags colliding in one slot together with their probabilities. The probabilities are calculated from a binomial distribution as explained in [21]:

$$
P_{s_{l}}(R)=\frac{\prod_{d=1}^{D}\left(\begin{array}{c}
R-\sum_{k}^{d-1} R_{k}^{c c} \\
R_{d}^{c c}
\end{array}\right) C !}{C^{R} \cdot(C-(D+U)) !} \prod_{e=2}^{R} \frac{1}{S(e) !},
$$

where $C$ is the number of postpreambles in a set (in our application, we selected $C=8$ ), $D$ denotes the number of 'colliding' colours and $R_{d}^{c c}$ represents the number of tags with the same colour for $d=1, \ldots, D$. Additionally, $U$ is the number of 'unique' colours. The values of $D, U$ and $R_{d}^{c c}$ for $d=1, \ldots, D$ are determined based on the corresponding scenario $s_{l}(l=1,2, \ldots, \mathrm{S}(\mathrm{R}))$ as detailed in Table 5 .

The remaining term, $S(e)$ is calculated as

$$
S(e)=\sum_{d=1}^{D} \mathbf{1}\left(R_{d}^{c c}=e\right), \quad e=1,2, \ldots, R .
$$

Here, $e=1, . ., R$ denotes the number of tags with the same colour and $\mathbf{1}(\cdot)$ is an indicator function:

$$
\mathbf{1}(x)= \begin{cases}1 & ; \text { if } x \text { is true } \\ 0 & ; \text { else }\end{cases}
$$

Take for example, six tags transmitting in one slot; then, $R=6$ and just one tag is having a distinct colour $U=1$, two tags are using the same colour $R_{1}^{c c}=2$, and three tags have an identical but different colour $R_{2}^{c c}=3$. Thus, the number of colliding colours is $D=2$. In Table 5 , this is scenario $6(3+2+1)$.

Each column of Table 5 lists various collision scenarios given a collision of $R$ tags, and each row of Table 5 represents a different collision scenario. In the first row of the table, scenario 1 is listed. Here, all tags involved in a collision have a different postpreamble. In the second row, we find scenario 2 , where two out of all colliding tags have the same postpreamble while others have a different one, and so on. Thus, for $R=2$ tags active in one slot, we can differentiate $S(R=2)=2$ scenarios; for $R=3$, the number of scenarios is $S(R=3)=3$; for $R=4$, the number is $S(R=4)=5$, and so on. The numbers in Table 5 represent the combination of tags with the same postpreamble. Indicated by the italic digit ' 1 ' are tags that can be successfully decoded due to their occurrence of a unique colour. Furthermore, the italic digit ' 2 ' denotes those tags with a single occurrence of the same postpreamble that can be decoded by the projection method (scenario 2) as explained in [21].

In Equation (4), we assumed that our reader resolves all tags in each slot in any collision scenario up to $R=M$ and acknowledges $J=M$ tags. For a reader that can detect and acknowledge only the italic parts of the collisions of Table 5 (we further on call this joint set the Cyan Set), we analytically obtain the following maximal theoretical throughput per slot:

$$
\operatorname{Tps}_{f}=\sum_{R=1}^{M} P_{R} \cdot\left(\sum_{l=1}^{S(R)} P_{s_{l}}(R) \cdot R_{s_{l}}^{\mathrm{sol}}(R)\right) .
$$

Here, $P_{R}$ is calculated as:

$$
P_{R}=\left(\begin{array}{l}
N \\
R
\end{array}\right)\left(\frac{1}{F}\right)^{R}\left(1-\frac{1}{F}\right)^{N-R}
$$

and represents the probability that exactly $R$ tags are active in one slot.

Now, we have taken into account the probability $P_{s_{l}}(R)$ (as listed in Table 5) of each scenario (from scenario $s_{1}$ up to $\left.s_{\mathrm{S}(\mathrm{R})}\right)$ and the number $R_{s_{l}}^{\text {sol }}(R)$ of tags that can be resolved in each scenario $s_{l}, l=1,2, . ., S(R)$ (joint set 
Table 5 Collision scenarios for up to eight colliding tags per slot

\begin{tabular}{|c|c|c|c|c|c|c|c|c|}
\hline Scenarios & $R=1$ & $R=2$ & $R=3$ & $R=4$ & $R=5$ & $R=6$ & $R=7$ & $R=8$ \\
\hline \multirow[t]{3}{*}{ Unique } & $P_{s 1}=1$ & $P_{S 1}=0.875$ & $P_{s 1}=0.656$ & $P_{s 1}=0.410$ & $P_{s 1}=0.205$ & $P_{s 1}=0.077$ & $P_{s 1}=0.019$ & $P_{s 1}=0.002$ \\
\hline & 1 & $1+1$ & $1+1+1$ & $1+1+1+1$ & $1+1+1+1$ & $1+1+1+1$ & $1+1+1+1$ & $1+1+1+1$ \\
\hline & & & & & 1 & $1+1$ & $1+1+1$ & $1+1+1+1$ \\
\hline \multirow[t]{41}{*}{ Mixed scenario } & & $P_{s 2}=0.125$ & $P_{s 2}=0.328$ & $P_{s 2}=0.492$ & $P_{s 2}=0.513$ & $P_{s 2}=0.385$ & $P_{s 2}=0.202$ & $P_{s 2}=0.067$ \\
\hline & & 2 & $2+1$ & $2+1+1$ & $2+1+1+1$ & $2+1+1$ & $2+1+1$ & $2+1+1$ \\
\hline & & & & & & $1+1$ & $1+1+1$ & $1+1+1+1$ \\
\hline & & & $P_{s 3}=0.016$ & $P_{s 3}=0.041$ & $P_{s 3}=0.154$ & $P_{s 3}=0.288$ & $P_{s 3}=0.337$ & $P_{s 3}=0.252$ \\
\hline & & & 3 & $2+2$ & $2+2+1$ & $2+2+1$ & $2+2+1$ & $2+2+1$ \\
\hline & & & & & & 1 & $1+1$ & $1+1+1$ \\
\hline & & & & $P_{s 4}=0.055$ & $P_{54}=0.103$ & $P_{s 4}=0.019$ & $P_{s 4}=0.084$ & $P_{s 4}=0.168$ \\
\hline & & & & $3+1$ & $3+1+1$ & $2+2+2$ & $2+2+2$ & $2+2+2$ \\
\hline & & & & & & & 1 & $1+1$ \\
\hline & & & & $P_{55}=0.002$ & $P_{55}=0.017$ & $P_{55}=0.128$ & $P_{55}=0.112$ & $P_{55}=0.011$ \\
\hline & & & & 4 & $3+2$ & $3+1+1$ & $3+1+1$ & $2+2+2$ \\
\hline & & & & & & 1 & $1+1$ & 2 \\
\hline & & & & & $P_{56}=0.009$ & $P_{s 6}=0.077$ & $P_{s 6}=0.168$ & $P_{56}=0.067$ \\
\hline & & & & & $4+1$ & $3+2+1$ & $3+2+1$ & $3+1+1+1$ \\
\hline & & & & & & & 1 & $1+1$ \\
\hline & & & & & $P_{s 7}=2 \cdot 10^{-4}$ & $P_{s 7}=0.002$ & $P_{S 7}=0.017$ & $P_{57}=0.224$ \\
\hline & & & & & 5 & $3+3$ & $3+2+2$ & $3+2+1$ \\
\hline & & & & & & & & $1+1$ \\
\hline & & & & & & $P_{58}=0.019$ & $P_{s 8}=0.112$ & $P_{58}=0.084$ \\
\hline & & & & & & $4+1+1$ & $3+3+1$ & $3+2+2$ \\
\hline & & & & & & & & 1 \\
\hline & & & & & & $P_{59}=0.003$ & $P_{s 9}=0.028$ & $P_{s 9}=0.028$ \\
\hline & & & & & & $4+2$ & $4+1+1$ & $3+3+1$ \\
\hline & & & & & & & 1 & 1 \\
\hline & & & & & & $P_{s 10}=0.001$ & $P_{s 10}=0.017$ & $P_{s 10}=0.006$ \\
\hline & & & & & & $5+1$ & $4+2+1$ & $3+3+2$ \\
\hline & & & & & & $P_{s 11}=3 \cdot 10^{-5}$ & $P_{s 11}=0.001$ & $P_{s 11}=0.028$ \\
\hline & & & & & & 6 & $4+3$ & $4+1+1$ \\
\hline & & & & & & & & $1+1$ \\
\hline & & & & & & & $P_{s 12}=0.003$ & $P_{s 12}=0.042$ \\
\hline & & & & & & & $5+1+1$ & $4+2+1$ \\
\hline & & & & & & & & 1 \\
\hline & & & & & & & $P_{s 13}=6 \cdot 10^{-4}$ & $P_{s 13}=0.004$ \\
\hline & & & & & & & $5+2$ & $4+2+2$ \\
\hline & & & & & & & $P_{s 14}=2 \cdot 10^{-4}$ & $P_{S 14}=0.006$ \\
\hline & & & & & & & $6+1$ & $4+3+1$ \\
\hline & & & & & & & $P_{s 15}=4 \cdot 10^{-6}$ & $P_{s 15}=1 \cdot 10^{-4}$ \\
\hline & & & & & & & 7 & $4+4$ \\
\hline & & & & & & & & $P_{s 16}=0.006$ \\
\hline & & & & & & & & $5+1+1+1$ \\
\hline & & & & & & & & $P_{s 17}=0.003$ \\
\hline
\end{tabular}






Collision scenarios for up to eight tags colliding in the same slot are listed in Table 5 . Here, the numbers in italics of the form $2+1+1 \ldots$ represent the number of tags with the same postpreamble. Italic digit ' $l$ ' indicates single occurrences of colours; italic digit ' 2 ' indicates single pairs occurring.

of italic numbers from corresponding rows of Table 5). Theoretically expected values of the throughput per slot are shown in Figure 5. The throughput curves follow with very good agreement a simple function $\operatorname{Tps}_{f}(F / N)$ (see Appendix 3 for more details). Values of the maximal theoretical throughput per slot together with the optimal frame size normalized to the tag population size are shown in Table 6 for $N_{\mathrm{RA}}=\{1,2,4\}$ receive antennas allowing for collision recovery factors of $M=\{1,2,4,8\}$.

Comparing Tables 3 and 6, we observe that for higher values of the collision recovery factor $M$, the loss in Tps performance increases because the number of unresolved tags becomes much higher.

\section{Collision recovery procedure}

Let us first consider an example by which we will explain how the proposed successive interference canceller with the projection concept typically works. In case when $R=$ 4 tags are colliding and two colliding tags have the same colour while the others have different unique colours (scenario 2), the vector form of the signals received by $N_{\mathrm{RA}}=$ 2 antennas [21] is as follows:

$$
\left[\begin{array}{l}
\mathbf{r}_{1}^{p p}(t) \\
\mathbf{r}_{2}^{p p}(t)
\end{array}\right]=\left[\begin{array}{llll}
h_{1,1} & h_{1,2} & h_{1,3} & h_{1,4} \\
h_{2,1} & h_{2,2} & h_{2,3} & h_{2,4}
\end{array}\right]\left[\begin{array}{l}
\mathbf{p}_{a} \\
\mathbf{p}_{b} \\
\mathbf{p}_{c} \\
\mathbf{p}_{c}
\end{array}\right]+\left[\begin{array}{l}
\mathbf{n}_{1}(t) \\
\mathbf{n}_{2}(t)
\end{array}\right]
$$

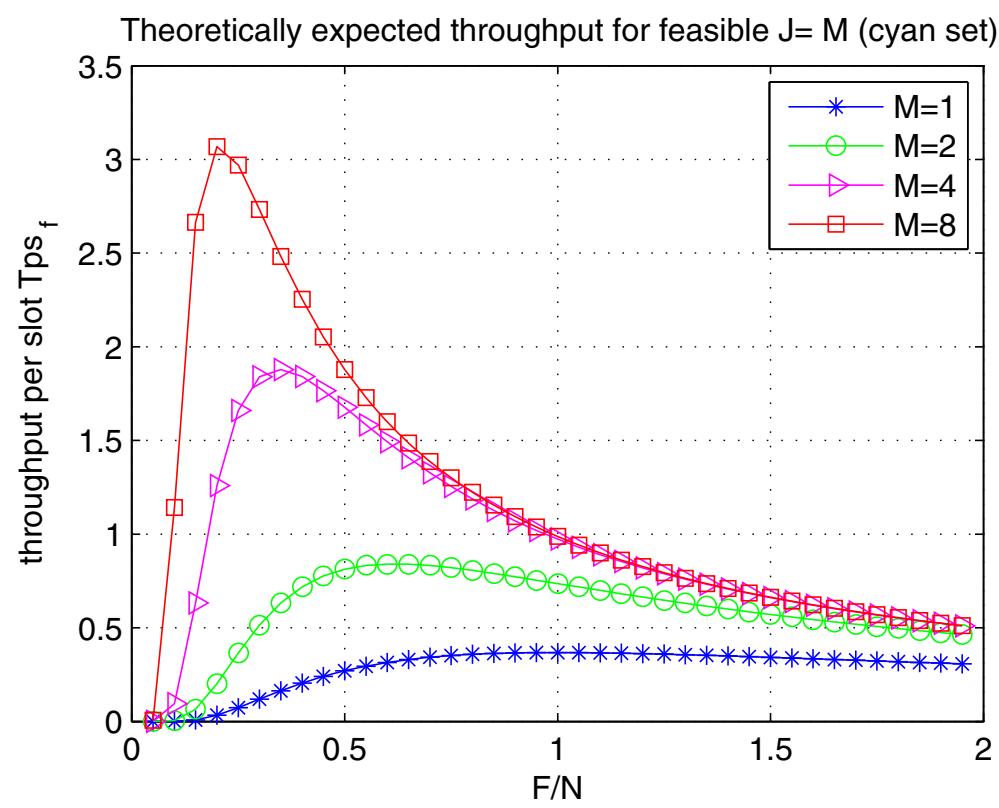

Figure 5 Theoretically expected throughput per slot curves for covered scenarios. Expected throughput as a function of slots per tag population $\mathrm{F} / \mathrm{N}$ for $J=M=2 N_{\mathrm{RA}}$ acknowledgements in the Cyan Set following Equation (10). 
Table 6 Maximal theoretical throughput for covered scenarios

\begin{tabular}{lccc}
\hline System & $\boldsymbol{F}_{\text {opt }} / \boldsymbol{N}$ & Tps & $\boldsymbol{R}_{\text {Tps }}$ \\
\hline$M=1 J=1$ & 1 & 0.368 & 1.000 \\
$M=2 J=2$ & 0.618 & 0.841 & 2.285 \\
$M=4 J=4$ & 0.345 & 1.879 & 5.106 \\
$M=8 J=8$ & 0.207 & 3.073 & 8.351 \\
\hline
\end{tabular}

The table shows optimal ratio $F_{\text {opt }} / N$, maximal theoretical throughput for covered scenarios and its relative improvement in the Cyan Set (see Appendix 3 for more details).

where $\mathbf{r}_{1}^{p p}(t)$ and $\mathbf{r}_{2}^{p p}(t)$ are parts of the received signals containing the postpreamble from antennas 1 and 2, respectively.

In this scenario, tags 3 and 4 share the same postpreamble, $\mathbf{p}_{c}$, and we cannot use an LS channel estimation technique from [12]. To overcome this situation, we propose a collision recovery procedure that consists of two phases. The first phase is performed by a successive interference cancellation (SIC), and the second is a projection of the constellation into the orthogonal subspace of the interference.

\subsection{Successive interference cancellation}

During this phase, we take out the signals from the tags with a unique postpreamble. Here, we assume that colliding tags are perfectly synchronized. The block diagram of the SIC architecture is shown in Figure 6.

The SIC begins with channel estimation according to Equation (3). Since these estimates are not correct for the tags with the same postpreamble, we use them just as auxiliary results. First, we cancel the signal from the strongest tag with unique postpreamble. This signal corresponds to the strongest channel coefficient and is found as the maximum among each columns of $\|\hat{\mathbf{H}}\|_{F}^{2}$, where $\|\cdot\|_{F}^{2}$ denotes the Frobenius norm. Through this, we ignore the signals from tags with the same postpreambles [21]. Additionally, we extract the tag modulation signals $\hat{\mathbf{a}}(t)$ with the MMSE receiver from Equation (2). Furthermore, we remodulate the signal from the strongest tag $j$ and subtract it from the received signal [21]:

$$
\overline{\mathbf{r}}(t) \leftarrow \overline{\mathbf{r}}(t)-\hat{\mathbf{h}}_{\mathbf{j}} \hat{a}_{j}(t)
$$

Here, $\hat{\mathbf{h}}_{\mathbf{j}}=\left[\hat{h}_{1 j}, . ., \hat{h}_{i j}, . ., \hat{h}_{N_{\mathrm{RA}}}\right]^{T}$ is the column vector of channel coefficients between the reader, the strongest tag $j$ and the receiving antennas $i=1,2, . ., N_{\mathrm{RA}}$.

Through this procedure, the received signal is cleaned from the influence of the strongest tag with its unique postpreamble, and the channel coefficients that correspond to this tag are stored. The new signal, $\overline{\mathbf{r}}(t)$, together with the new set $S_{M}$ of postpreambles without the postpreamble that corresponds to the strongest tag signal, is used as an input signal for the next iteration of the SIC.

In-phase/quadrature (IQ) diagrams of a received signal at antennas 1 and 2, when four tags are colliding, are presented in Figure 7. Here, it is difficult to detect states due to the superposition of many tag signals. During the successive interference cancellation, the signals from tags with a unique postpreamble are taken out. The remaining signal, after SIC, consists of the signals from the tags with the same postpreamble, tags 3 and 4, whose IQ diagrams are shown in Figure 8. The channel coefficients that correspond to this signals are estimated by a projection of the constellation into the orthogonal subspace of the interference from [10].

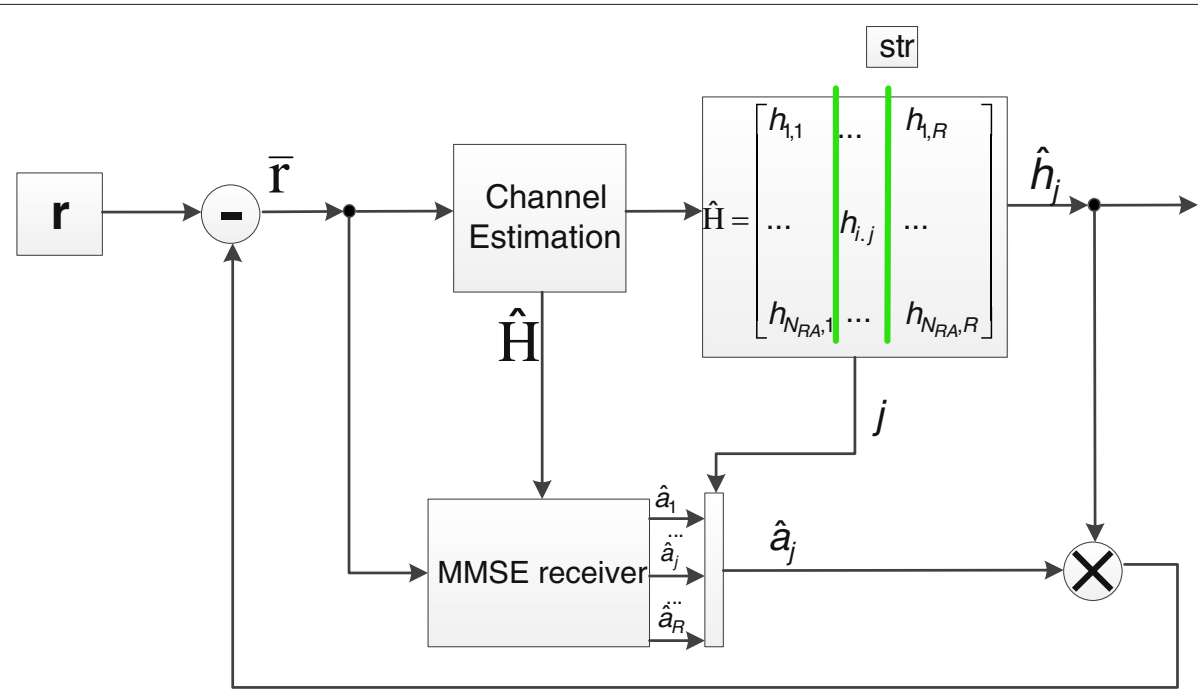

Figure 6 Block diagram of successive interference cancellation architecture. 


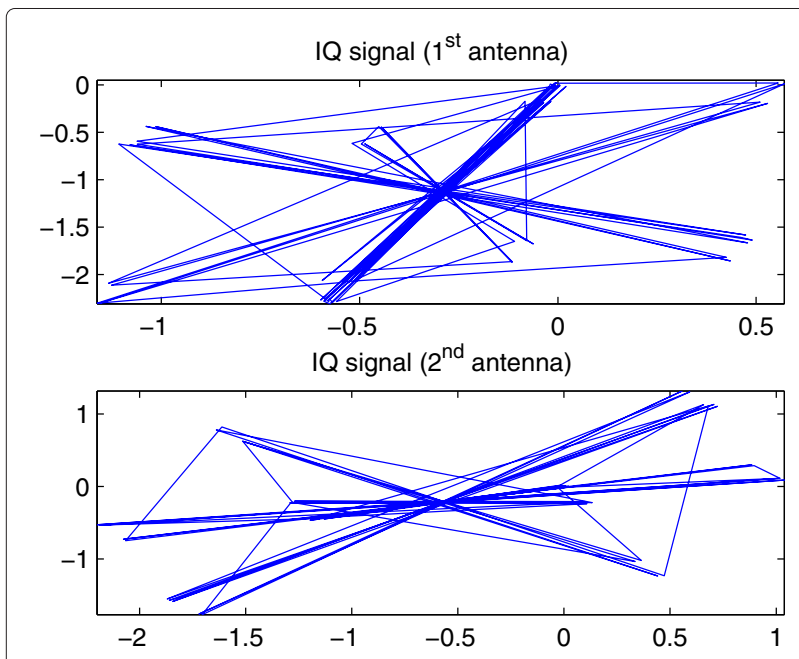

Figure 7 IQ diagram of the received signal from tags 1 to 4 at antennas 1 and 2.

\subsection{Channel estimation with projections}

After SIC, the remaining signal is composed of signals that originate from two tags. Thus, theoretically, we should be able to differentiate $2^{R=2}=4$ states in the IQ diagram of Figure 8: the state when both tags are absorbing $\hat{C}_{i}^{a, a}$, the state when both tags are reflecting $\hat{C}_{i}^{r, r}$ and the states in which one tag is absorbing and one is reflecting $\hat{C}_{i}^{a, r}, \hat{C}_{i}^{r, a}$ [21].

The tag response to the reader's Query command begins with the preamble, according to the EPCglobal standard for UHF RFID [1]. During the preamble, both tags modulate the same bits 0 and 1 (subspace $\mathcal{C}_{r}$ ), and the reflecting state is estimated [21]:

$$
\hat{C}_{i}^{r, r}=\max \left\{r_{i}[k]\right\}_{t_{1 b i t}} .
$$

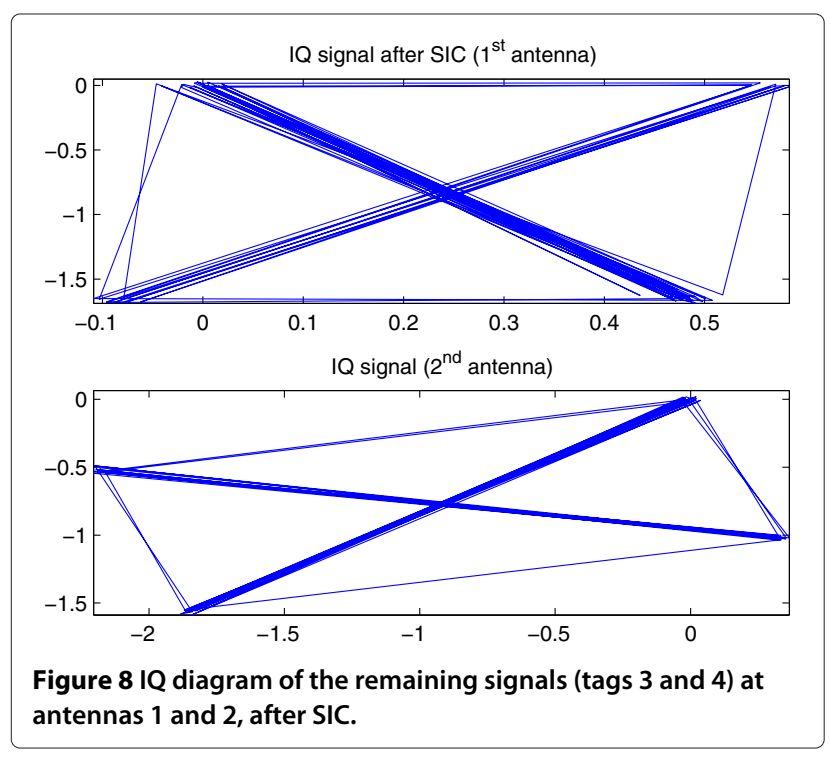

Here, $r_{i}[k]$ is the sample of the received signal from $i$ th antenna taken within the duration of the first preamble bit $t_{1 \text { bit. }}$

The absorbing state is determined by averaging the received signal over the time period $T$ before the tag response $\hat{C}_{i}^{a, a}=E\left\{r_{i}[k]\right\}_{T}$.

The remaining states occur when tags modulate different data during an RN16 and are estimated as the points with the maximal signal strength in a subspace orthogonal to $\mathcal{C}_{r}[10,21]$ :

$$
\hat{C}_{i}^{a, r}=\max _{k}\left\{r_{i \perp}[k]\right\}, \quad \hat{C}_{i}^{r, a}=\min _{k}\left\{r_{i \perp}[k]\right\},
$$

where $r_{i \perp}[k]$ is the signal component located in the orthogonal subspace.

Using the fact that the modulation signals are perfect on-off keying, the remaining channel coefficients are as follows:

$$
\hat{h}_{i, 1}=\hat{C}_{i}^{r, r}-\hat{C}_{i}^{a, r}, \quad \hat{h}_{i, 2}=\hat{C}_{i}^{r, r}-\hat{C}_{i}^{r, a} .
$$

With this, we have finished the channel estimation process, and the tag signals are extracted by the MMSE receiver from Equation (2).

Generally, the remaining signal after SIC is composed of the signals from tags with the same postpreambles. However, this signal is disturbed by the channel, noise and accumulated errors during the SIC process. As the disturbances are higher, there are more intersections, leading to a less accurate channel estimation.

\section{Performance analysis}

The performance analysis is achieved through Monte Carlo simulations. In the simulated system, the RFID reader has one transmitting and up to four receiving antennas $N_{\text {RA. }}$. In a first experiment to validate our predicted performance metrics, the number $R$ of tags that are active in one slot is kept fixed and can be set from one, i.e. transmission without collision, to up to eight, when eight tags are colliding in one slot. The channel between the reader's transmit antenna, the tag and the receiving antenna of the reader is modelled as a double Rayleigh fading channel as in [10]. Furthermore, imperfections like additive noise, an imperfect channel estimation, errors accumulated during successive interference cancellation and a non-ideal projection of the constellation into the orthogonal subspace of interference are assumed in this simulator. Additionally, we assume that colliding tags are perfectly synchronized and that the reader can exactly determine the size of the tag population. The influence of such parameters is left to be investigated in the future work.

As a performance measure, we observe the bit error ratio (BER) and the average number of successfully received packets (NSRP) per slot for different levels of 
average signal-to-noise ratios (SNRs). The SNR is averaged over the receiving antennas as explained in [11]. In NSRP calculations, the part of the signal that contains the RN16 number is considered as one packet. The NSRP is averaged over $N_{\text {iter }}=50 \cdot 10^{\frac{5 \mathrm{NN}[d B]}{10}}+50$ iterations [21], guaranteeing approximately the same quality (confidence interval) in the results. Around each point in the BER figures, a confidence interval that contains $95 \%$ of the obtained results is plotted to evaluate the quality of the simulations.

In the following, we present simulation results for an extreme scenario in which eight tags per slot are colliding but in which two tags have identical colour and the remaining six tags have unique colours $(2+1+1+1+1+1+1)$ so that we can expect them to resolve. In Figures 9, 10, 11, 12, 13 and 14, the results for the MMSE receiver with up to four receiving antennas $\left(N_{\mathrm{RA}}=1,2,3,4\right)$ are shown. Figures 9 and 12 represent BER and NSRP results, respectively, obtained by receivers with perfect channel knowledge. For these receivers, we do not perform channel estimation, and the postpreamble distribution does not have any influence. Thus, there is no decrease in the system performance, and we will use them as reference to the highest achievable performance of the designed system.

Results obtained with the receivers that apply an LS channel estimation method instead are shown in Figures 10 and 13, for BER and NSRP, respectively. It can be observed that since two out of eight colliding tags have the same postpreamble, the channel cannot be estimated correctly by a standard LS estimator, and the MMSE receiver cannot recover from this collision. The BER curves are saturating at high values. Nevertheless, some packets are decoded correctly as the NSRP curves show that in average more than four packets can be correctly received with four antenna receivers $\left(N_{\mathrm{RA}}=4\right)$. Hence, the errors are mostly concentrated in packets from tags with the same postpreamble, while the packets from the other six tags are less affected.

In Figures 11 and 14, the results obtained from the proposed smart receiver with the two-phase channel estimation and collision recovery are presented. Even though the BER values are significantly higher when compared to the reader with perfect channel knowledge, the performances in NSRP are comparable. It can be observed that a reader with four receiving antennas can successfully recover from this collision and in average can successfully receive almost seven packages. Corresponding BER and NSRP figures in the case of four tags transmitting simultaneously $(2+1+1)$ are presented and discussed in [21].

As an additional performance measure, the throughput per slot for different levels of the average SNR is investigated. The throughput per slot is calculated for all tags that a reader with $i$ receive antennas and collision recovery factor $M$ can resolve from a collision $(R=$ $1, \ldots, M)$. The probability that exactly $R$ tags are active in one slot is taken into account. Furthermore, for each number of colliding tags $R$, the corresponding probabilities of solvable scenarios, listed in Table 5 , are also

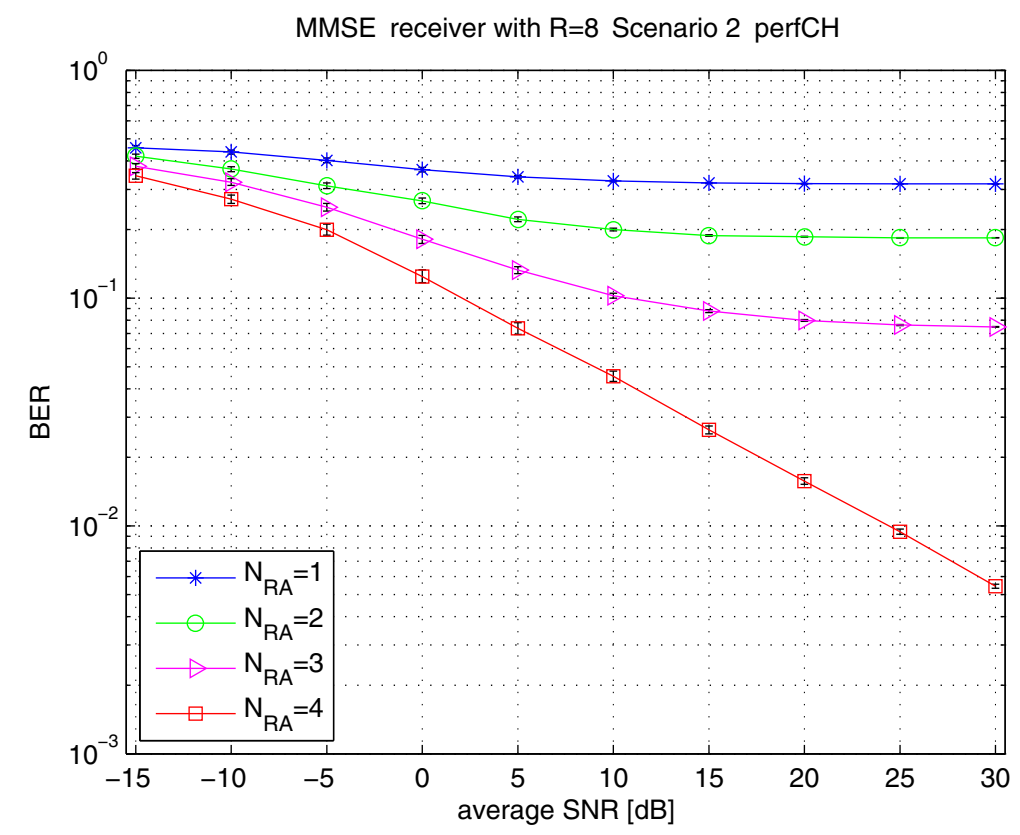

Figure 9 BER over SNR for MMSE receivers $(R=8$; perfect channel LS, $2+1+1+1+1+1+1)$. 




Figure 10 BER over SNR for MMSE receivers $(R=8$; estimated channel LS, $2+1+1+1+1+1+1)$.

included. All solvable scenarios are simulated separately and averaged over $N_{\text {iter }}$ iterations. The results obtained from simulation are multiplied with the corresponding scenario probability and with the corresponding probability that exactly $R$ tags are active in one slot. By combining all individual collision scenarios, the throughput per slot can be calculated semi-analytically as a function of SNR:

$$
\begin{gathered}
\operatorname{Tps}_{\mathrm{FSA}, i}(\mathrm{SNR})=\sum_{R=1}^{M} P_{R} \cdot\left(\sum_{l=1}^{S(R)} P_{S_{l}}(R) \cdot \mathrm{Sd}_{i, R}^{s_{l}}(\mathrm{SNR})\right), \\
i=1,2, . ., N_{\mathrm{RA}}
\end{gathered}
$$



Figure 11 BER over SNR for MMSE receivers $(R=8$; estimated channel SIC and the projection, $2+1+1+1+1+1+1)$. 


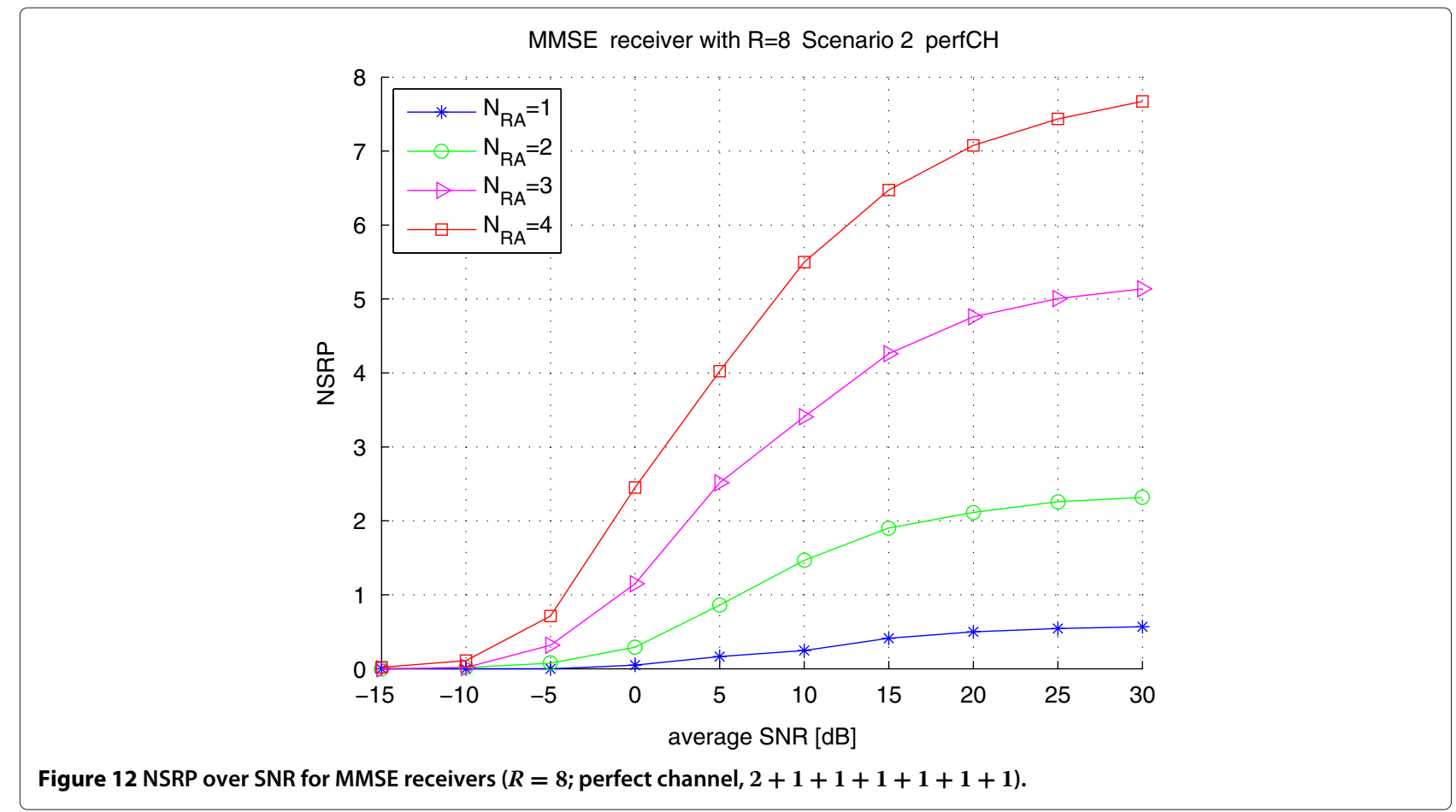

where $\mathrm{Sd}_{i, R}^{s_{l}}(\mathrm{SNR})$ denotes the average number of successfully decoded tags in a system with $i$ receive antennas in scenario $s_{l}, l=1,2, . ., S(R)$. Variable $\mathrm{Sd}_{i, R}^{s_{l}}(\mathrm{SNR})$ represents the average number of packets in a slot that are correctly received, and at best, it is close to $R_{s_{l}}^{\text {sol }}(R)$. The values of $\mathrm{Sd}_{i, R}^{s_{l}}(\mathrm{SNR})$ are taken from simulations and are thus dependent on the SNR. In Equation (16), $P_{R}$ represents the probability that exactly $R$ tags are active in one slot, and this probability is calculated based on Equation (11) for the optimal value $F_{\text {opt }}$ taken from Table 6 . The

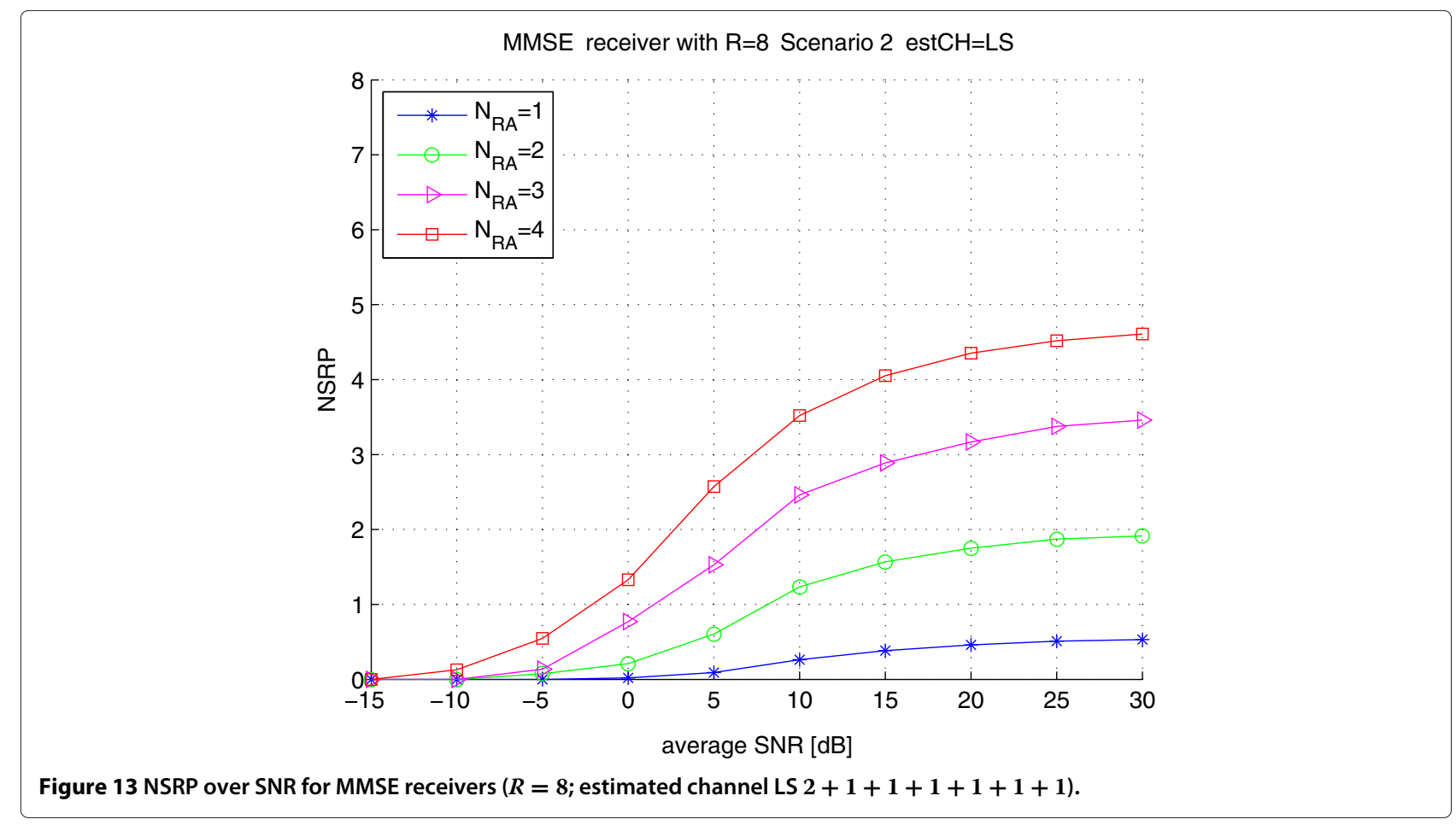




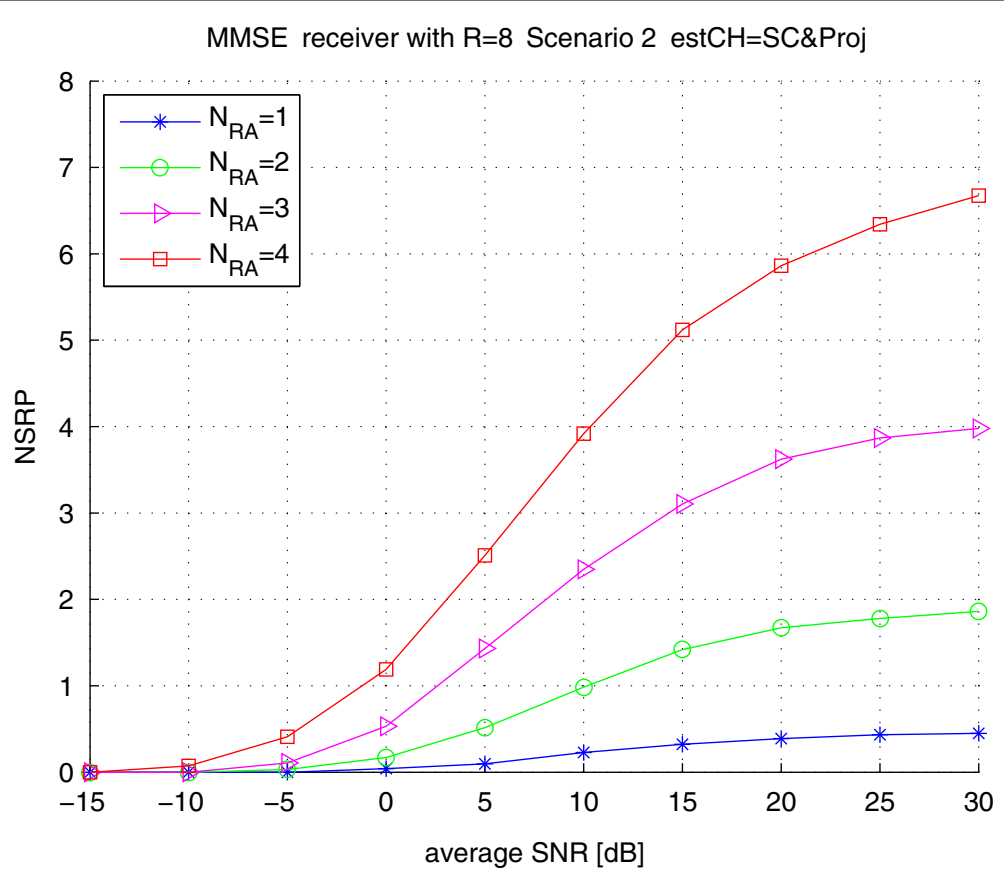

Figure 14 NSRP over SNR for MMSE receivers $(R=8$; estimated channel SIC and the projection, $2+1+1+1+1+1+1)$.

probabilities of scenarios $P_{S_{1}}, \ldots, P_{S_{S(R)}}$ are taken from Table 5.

In the case of perfect channel knowledge, the collision scenarios are irrelevant, and thus, we obtain the even simpler semi-analytical form [21]:

$$
\operatorname{Tps}_{\mathrm{FSA}, i}^{\mathrm{opt}}(\mathrm{SNR})=\sum_{R=1}^{M} P_{R} \cdot \mathrm{Sd}_{i, R}(\mathrm{SNR}) .
$$

Here, the probability $P_{R}$ is calculated according to Equation (11) for $F_{\text {opt }}$ taken from Table 3, and the values of $\mathrm{Sd}_{i, R}(\mathrm{SNR})$ correspond to those of $\mathrm{Sd}_{i, R}^{s_{l}}(\mathrm{SNR})$ but are now obtained from simulations with perfect channel knowledge.

A comparative overview of the expected throughput per slot for the MMSE receiver with a different number of receiving antennas in case of up to eight tags transmitting in one slot is presented in Figure 15. In our simulations, we included all cases from $R=1$ to $R=8$ collisions, which are equivalent to $93.5 \%$ of all cases for the given value of the optimal frame size $F_{\text {opt }}$. Our simulation results in Figure 15 reflect very well that we are only able to resolve $74.3 \%$ of these cases. The missing $6.5 \%$ of collisions larger than $R=8$ would not change the results considerably. The maxima of the theoretically expected throughputs based on perfect channel knowledge are indicated by the horizontal solid lines according to Equation (4), also listed in Table 3 (third column Tps), while the maxima of the theoretically expected throughputs from postpreamble-based channel estimation are represented by the dotted horizontal lines according to Equation (10), also listed in the third column Tps of Table 6. In Figure 15, the receivers with perfect channel knowledge are represented by solid curves (obtained based on Equation (17)), and the proposed receivers with postpreamble-based channel estimation in the Cyan Set are represented by dotted curves (obtained based on Equation 16). In both cases, the corresponding groups of curves are approaching their theoretical maxima very well. At high SNR, the simulated curves attain their theoretical maxima from Equation (4) (the case with perfect channel knowledge) and from Equation (10) (the case with postpreamble-based channel estimation). While the curves for single antenna systems with $M=1$ and $M=2$ are exhibiting the same performance with postpreamble-based channel estimation as with perfect channel knowledge, for two antennas, there is a slight loss visible, and for $N_{\mathrm{RA}}=4$ antennas, the loss due to channel estimation is quite pronounced. It can be observed that at a high SNR of $30 \mathrm{~dB}$, the throughput per slot increases dramatically when compared to the conventional reader even though the performance is hampered by channel estimation losses. In the case of a receiver with channel estimation and collision recovery factor $M=8$, the throughput per slot is still more than eight times higher than that of a conventional system (plotted with the blue curve). Even though we have not covered all of the collision scenarios with the proposed receiver but just the Cyan Set, the obtained results are quite satisfactory. 




Figure 15 Throughput in the case of perfect channel knowledge vs. throughput based on postpreamble channel estimation in Cyan Set. Continuous horizontal lines are bounds according to Equation (4), and dashed horizontal lines are following Equation (10).

Based on Figure 15, we observe the Tps for the different values of SNR, and according to that, we recalculate how many tags are left for the next frame in the same inventory round. For a receiver with collision recovery factor $M=8$, for example, the Tps value at the $\mathrm{SNR}=15 \mathrm{~dB}$ is $\mathrm{Tps}^{15 \mathrm{~dB}}=2.805$. The theoretically expected maximal throughput per slot is $\operatorname{Tps}_{f}=3.073$ for $F_{\text {opt }} / N=0.207$ (Table 6). Taking into account the optimal frame duration, the average number of tags that are successfully decoded within the duration of the first frame is $N^{\text {dec }}=$ round $\left(\mathrm{Tps}^{15 \mathrm{~dB}} \cdot F_{\mathrm{opt}}\right)=581$. Thus, for the next inventory round, $N^{\text {left }}=419$ tags are left. On the other hand, a conventional receiver has a maximal throughput per slot Tps $=0.368$ for a frame size equal to the tag population size $F_{\text {opt }} / N=1$, and at the $\mathrm{SNR}=15 \mathrm{~dB}, \mathrm{Tps}^{15 \mathrm{~dB}}=$ 0.212 . Accordingly, within the first frame duration, a conventional receiver successfully reads out $N^{\mathrm{dec}}=212$ tags, and $N^{\text {left }}=N-N^{\text {dec }}=788$ tags are left for the next round.

Assuming that the reader recalculates optimal frame sizes for the residual tag population $N^{*}=N^{\text {left }}$ before announcing the next frame, the following Figures 16 and 17 are obtained. For the new tag population, the optimal frame size is calculated according to the ratio from Table 6 , and $N^{\text {dec }}=$ round $\left(\mathrm{Tps}^{15 \mathrm{~dB}} \cdot F_{\text {opt }}\right)$. The new residual tag population is $N^{\text {left }} \leftarrow N^{*}-N^{\text {dec }}$ and so on.

The number of residual tags, tags that still have not been decoded, versus the number of elapsed slots is shown in Figure 16. For the reader with collision recovery factor
$M=1$, a number of 4,693 slots (22 frames) are necessary to decode $99.5 \%$ of the tags in the reader range. A reader with collision recovery factor $M=2$ needs 2,447 slots (19 frames) to decode $99.6 \%$, while the readers with higher collision recovery factors are much faster. The reader that is capable of recovering from a collision of up to four tags active in a slot $M=4$ and acknowledges all of them spends 775 slots (19 frames) for successfully decoding $99.6 \%$, and the reader with $M=8$ decodes $99.7 \%$ of tags in just 355 slots (7 frames).

In Figure 17, the number of decoded tags versus the frame index is shown for different receivers. These values are also listed in Table 7 along with the optimal frame sizes $F_{\text {opt }}$.

In Table 8, the necessary number of slots is presented for reading a fixed percentage of the tag population together with the relative improvement compared to the conventional reader with collision recovery factor $M=1$. In the case of our smart collision recovery reader receiver, the relative improvement is larger than 13 times at a realistic SNR of $15 \mathrm{~dB}$. As compared to the throughput increase at $\mathrm{SNR}=30 \mathrm{~dB}$, the relative improvement at $\mathrm{SNR}=$ $15 \mathrm{~dB}$ is much higher and in accordance with Figure 15 as expected.

\section{Conclusion}

In this work, we have analysed the theoretical throughput of an FSA RFID system. We have studied the influence of several parameters on the system throughput, and we 




Figure 16 Number of residual tags vs. number of used slots.

found the maxima of the theoretically expected throughput for receivers with different collision recovery factors and for different receiver architectures. We have investigated the benefits of an additional postpreamble to the throughput, and we observed that without taking into account the receiver structure and the channel estimation method, it is possible to increase the system throughput by more than 17 times. On the other hand, if the receiver structure is taken into account, then a throughput increase of more than 12 times can still be achieved for a reader capable of successfully reading and acknowledging up to eight tags per slot. In these calculations, it is assumed that the readers have perfect channel knowledge. However, in order to recover from a collision, a reader needs to perform channel estimation. For the channel estimation procedure, the tags are augmented by postpreambles. Based on this, we can differentiate several collision scenarios, and if we include the probability of scenarios


Figure 17 Number of decoded tags vs. number of frames. 
Table 7 Number of decoded tags and optimal frame duration at SNR $=15 \mathrm{~dB}$

\begin{tabular}{|c|c|c|c|c|c|c|c|}
\hline \multicolumn{2}{|c|}{$M=1$} & \multicolumn{2}{|c|}{$M=2$} & \multicolumn{2}{|c|}{$M=4$} & \multicolumn{2}{|c|}{$M=8$} \\
\hline$F_{\mathrm{opt}}$ & $N^{\text {dec }}$ & $F_{\mathrm{opt}}$ & $N^{\text {dec }}$ & $F_{\text {opt }}$ & $N^{\text {dec }}$ & $F_{\mathrm{opt}}$ & $N^{\mathrm{dec}}$ \\
\hline 1,000 & 212 & 618 & 252 & 345 & 455 & 207 & 581 \\
\hline 788 & 167 & 462 & 188 & 188 & 248 & 87 & 244 \\
\hline 621 & 131 & 346 & 141 & 102 & 134 & 36 & 101 \\
\hline 490 & 104 & 259 & 105 & 56 & 74 & 15 & 42 \\
\hline 386 & 82 & 194 & 79 & 31 & 41 & 7 & 20 \\
\hline 304 & 64 & 145 & 59 & 17 & 22 & 2 & 6 \\
\hline 240 & 51 & 109 & 44 & 9 & 12 & 1 & 3 \\
\hline
\end{tabular}

that can be resolved and the number of tags that can be successfully decoded, the maximal theoretical throughput is still about eight times the throughput of a conventional system at $30 \mathrm{~dB}$ SNR. For theoretical calculations, it is assumed that the reader can successfully decode all tags with unique postpreambles, regardless of the scenario, and also all tags colliding in scenario 2, where exactly two of colliding tags share an identical postpreamble.

Practically, for recovering from scenario 2 collisions, we propose a two-phase collision recovery procedure. In the first phase, the signal part from the tags with unique postpreambles is taken out during a successive interference cancellation process, while in the second phase, the remaining signal, originating from the tag pair with the same postpreambles, is resolved using the projection of the constellation into the orthogonal subspace of the interference. The so obtained results show that the proposed method provides satisfactory results.

Moreover, the necessary time to read out a high tag population is investigated. We showed that at the average SNR of $15 \mathrm{~dB}$, a smart reader with collision recovery factor $M=8$ successfully decodes all tags in the reader range, more than 13 times faster than a conventional reader, and performances are considerably enhanced.

Table 8 Number of slots spent for decoding $95 \%, 98 \%$ and $\mathbf{9 9 . 5} \%$ and relative improvement at $\mathrm{SNR}=15 \mathrm{~dB}$

\begin{tabular}{lcccc}
\hline $\begin{array}{l}\text { Slot } \\
\text { Relative improvement }\end{array}$ & $\boldsymbol{M = 1}$ & $\boldsymbol{M = 2}$ & $\boldsymbol{M = 4}$ & $\boldsymbol{M = 8}$ \\
\hline $95 \%$ & 4481 & 2334 & 720 & 338 \\
& 1 & 1.920 & 6.224 & 13.257 \\
$98 \%$ & 4623 & 2408 & 743 & 349 \\
& 1 & 1.920 & 6.222 & 13.246 \\
$99.5 \%$ & 4693 & 2445 & 754 & 354 \\
& 1 & 1.920 & 6.224 & 13.257 \\
\hline
\end{tabular}

The table shows the number of slots spent for decoding for decoding $95 \%, 98 \%$ and $99.5 \%$ and the relative improvement at $\mathrm{SNR}=15 \mathrm{~dB}$ for $N_{\mathrm{RA}}=\{1,2,4\}$ antennas and corresponding collision recovery factor $M=\{1,2,4,8\}$.

\section{Appendix 1}

\section{Approximation of $\operatorname{Tps}_{M}$}

The number of tags in the reader range $N$ is much bigger than the number of colliding tags $R$ that our reader is capable of resolving $(N \gg R)$. Taking this into account, the first part of Equation (4)

$$
\left(\begin{array}{l}
N \\
R
\end{array}\right)=\frac{N \cdot(N-1) \cdot \ldots \cdot(N-R+1)}{R !}
$$

can be approximated by

$$
\left(\begin{array}{l}
N \\
R
\end{array}\right) \approx \frac{N^{R}}{R !}
$$

The part $\left(1-\frac{1}{F}\right)^{N-R}$ for $F \gg 1$ can be approximated by

$$
\begin{aligned}
\left(1-\frac{1}{F}\right)^{N-R} & \approx\left(\left(1-\frac{1}{F}\right)^{F}\right)^{\frac{N-R}{F}}=\underbrace{\left(\left(1+\frac{1}{-F}\right)^{-F}\right)^{\frac{N-R}{F}}}_{\approx e^{-1}} \\
& \approx e^{-\frac{N-R}{F}}
\end{aligned}
$$

Using that $N \gg R$, we finally can approximate Equation (4) by

$$
\begin{aligned}
\operatorname{Tps}_{M} & \approx \sum_{R=1}^{M} \frac{N^{R}}{R !}\left(\frac{1}{F}\right)^{R} e^{-\frac{N}{F}} R \\
& \approx \sum_{R=1}^{M}\left(\frac{N}{F}\right)^{R} e^{-\frac{N}{F}} \frac{1}{(R-1) !} \\
& \approx \sum_{R=1}^{M}\left(\frac{F}{N}\right)^{-R} e^{-\frac{1}{F / N}} \frac{1}{(R-1) !}
\end{aligned}
$$

Thus, the entire expression is a function of $F / N$.

If we approximate our functions now as functions of $x=$ $F / N$, they take on the form:

$$
\operatorname{Tps}_{M} \approx \sum_{R=1}^{M} x^{-R} e^{-\frac{1}{x}} \frac{1}{(R-1) !}
$$

which offers them to differentiate with respect to $x$ and to find the maxima for throughput and thus the optimal $F / N$ ratio.

The obtained optimal ratios $F / N$ for maximal throughput are $[1 ; 0.618 ; 0.441 ; 0.340 ; 0.275 ; 0.230 ; 0.197 ; 0.172]$ for $R=1,2, \ldots, 8$. When compared with simulation results, this approximation shows an almost perfect agreement. 


\section{Appendix 2}

\section{Approximation of $\mathrm{Tps}_{\mathrm{C}}$}

Following the approximations from Appendix 1 now for $\frac{N}{C} \gg R_{C}$ and $F \gg 1$, Equation (5) can be approximated by

$$
\operatorname{Tps}_{C} \approx C \sum_{R_{C}=1}^{J_{C}}\left(\frac{F}{N / C}\right)^{-R_{C}} e^{-\frac{1}{F C / N}} \frac{1}{\left(R_{C}-1\right) !}
$$

Furthermore, we can approximate this term now as a function of $x=F / N$ :

$$
\operatorname{Tps}_{C} \approx C \sum_{R_{C}=1}^{J_{C}}(C x)^{-R_{C}} e^{-C x} \frac{1}{\left(R_{C}-1\right) !} .
$$

This allows to differentiate with respect to $x$ and to find the maxima for throughput and thus the optimal $F / N$ ratio. When compared with simulation results, this approximation shows an almost perfect agreement.

\section{Appendix 3}

\section{Approximation of $\mathrm{Tps}_{f}$}

Using the approximations from Appendix 1, Equation (10) can be approximated by

$$
\operatorname{Tps}_{f} \approx \sum_{R=1}^{M}\left(\frac{F}{N}\right)^{-R} e^{-\frac{1}{F / N}} \frac{1}{R !} \cdot\left(\sum_{l=1}^{S(R)} P_{s_{l}}(R) \cdot R_{s_{l}}^{\mathrm{sol}}(R)\right)
$$

Thus, the entire expression can be written as a function of $x=F / N$.

$$
\operatorname{Tps}_{f} \approx \sum_{R=1}^{M} x^{-R} e^{-\frac{1}{x}} \frac{1}{R !} \cdot\left(\sum_{l=1}^{S(R)} P_{s_{l}}(R) \cdot R_{s_{l}}^{\mathrm{sol}}(R)\right)
$$

Now we can differentiate with respect to $x$ and find the optimal $F / N$ ratio that corresponds to the throughput maxima. When compared with simulation results, this approximation shows an almost perfect agreement.

\section{Competing interests}

The authors declare that they have no competing interests.

\section{Acknowledgements}

This work has been funded by the Christian Doppler Laboratory for Wireless Technologies for Sustainable Mobility and its industrial partner Infineon Technologies. The financial support by the Federal Ministry of Economy, Family and Youth and the National Foundation for Research, Technology and Development is gratefully acknowledged. We finally like to thank Christoph Mecklenbräuker for his fruitful discussions and stimulative thoughts.

Received: 14 November 2012 Accepted: 21 March 2013 Published: 30 April 2013

\section{References}

1. EPCGlobal, EPC radio-frequency identity protocols class-1 generation-2 UHF RFID (9 Nov 2010). http://www.gs1.org/gsmp/kc/epcglobal/ uhfc1g2/uhfc1g2_1_2_0-standard-20080511.pdf
2. MV Bueno-Delgado, J Vales-Alonso, FJ Gonzalez-Castao, in Proceedings of 35th Conf. IEEE Industrial Electronics Society (IECON). Analysis of DFSA anti-collision protocols in passive RFID environments (Porto, Portugal, 3-5 Nov 2009)

3. B Knerr, M Holzer, C Angerer, M Rupp, Slot-wise maximum likelihood estimation of the tag population size in FSA protocols. IEEE Trans. Commun. 58(2), 578-585 (2010)

4. J Yu, KH Liu, X Huang, G Yan, in Proceedings of International Conference on Microwave and Millimeter Wave Technology, 2008. ICMMT 2008. An anti-collision algorithm based on smart antenna in RFID system (Nanjing, China, 21-24 April 2008)

5. C Angerer, G Maier, MV Bueno-Delgado, M Rupp, J Vales-Alonso, in Proceedings of the IEEE International Conference on Industrial Technology (ICIT'10). Single antenna physical layer collision recovery receivers for RFID readers (Vina del Mar-Valparaiso, Chile, 14-17 Mar 2010)

6. A Bletsas, J Kimionis, AG Dimitriou, GN Karystinos, Single-antenna coherent detection of collided FMO RFID signals. IEEE Trans. Commun. 60(3), 756-766 (2012)

7. J Kimionis, A Bletsas, AG Dimitriou, GN Karystinos, in Proceedings of IEEE International Conference on RFID-Technologies and Applications (RFID-TA). Inventory time reduction in Gen2 with single-antenna separation of FM0 RFID signals (Sitges, Spain, 15-16 Sept 2011)

8. DD Donno, V Lakafosis, L Tarricone, MM Tentzeris, in Proceedings of IEEE MTT-S Int. Microwave Symp. Digest (MTT). Increasing performance of SDR-based collision-free RFID systems (Montreal, Canada, 17-22 June 2012)

9. S Kim, S Kwack, S Choi, BG Lee, in Proceedings of 17th Asia-Pacific Conf. Communications (APCC). Enhanced collision arbitration protocol utilizing multiple antennas in RFID systems (Kota Kinabalu, Malaysia, 2-5 Oct 2011)

10. C Angerer, R Langwieser, M Rupp, RFID reader receivers for physical layer collision recovery. IEEE Trans. Commun. 58(12), 3526-3537 (2010)

11. J Kaitovic, R Langwieser, M Rupp, in Proceedings of IEEE International Conference on RFID-Technologies and Applications (RFID-TA). RFID reader with multi antenna physical layer collision recovery receivers (Sitges, Spain, 15-16 Sept 2011)

12. J Kaitovic, M Šimko, R Langwieser, M Rupp, in Proceedings of 2012 IEEE International Conference on RFID (RFID). Channel estimation in tag collision scenarios (Orlando, Florida, 3-5 April 2012)

13. K Fyhn, RM Jacobsen, P Popovski, A Scaglione, T Larsen, Multipacket reception of passive UHF RFID tags: a communication theoretic approach. IEEE Trans Signal Process. 59(9), 4225-4237 (2011)

14. F Ricciato, P Castiglione, Pseudo-random Aloha for enhanced collision-recovery in RFID. CoRR. abs/1209.4763 (2012)

15. J Myung, W Lee, J Srivastava, Adaptive binary splitting for efficient RFID tag anti-collision. IEEE Commun. Lett. 10(3), 144-146 (2006)

16. Y Cui, in Proceedings of IEEE International Conference on RFID-Technologies and Applications (RFID-TA). System efficiency of collision recover binary tree algorithm in RFID (Nice, France, 5-7 Nov 2012)

17. A Lazaro, D Girbau, D Salinas, Radio link budgets for UHF RFID on multipath environments. IEEE Trans. antennas Propag. 57(4), 1241-1251 (2009)

18. D Kim, MA Ingram, WW Smith, Measurements of small-scale fading and path loss for long range RF tags. IEEE Trans. Antennas Propag. 51(8), 1740-1749 (2003)

19. JD Griffin, GD Durgin, Gains for RF tags using multiple antennas. IEEE Trans. Antennas Propag. 56(2), 563-570 (2008)

20. J Balakrishnan, M Rupp, $\mathrm{H}$ Viswanathan, in Proceedings of Conference on Multiaccess, Mobility and Teletraffic for Wireless Communications. Optimal channel training for multiple antenna systems (Florida, USA, 3-6 Dec 2000)

21. J Kaitovic, R Langwieser, M Rupp, in Proceedings of the 4th International EURASIP Workshop on RFID Technology. Advanced collision recovery receiver for RFID (Torino, Italy, 27-28 Sept 2012)

doi:10.1186/1687-3963-2013-7

Cite this article as: Kaitovic et al: A smart collision recovery receiver for RFIDs. EURASIP Journal on Embedded Systems 2013 2013:7. 